\title{
Detección de portadores sanos en la distrofia muscular progresiva
}

\section{4 familias}

DR. MARCElo DEVILAT B.***, DRA. MARTA COlOMBO C. ***, SRTA. TERESA BARROS H. ***. SRTA. LILIANA MEDINA M. *.

La Distrofia Muscular Progresiva Pseudohipertrófica tipo Duchenne (DMP), es la más frecuente de las Distrofias Musculares (1). Se distingue de ellas, por tener herencia recesiva ligada al sexo, por comenzar en la época preescolar, por tener una rápida progresión de los síntomas hasta un desenlace fatal y por el aumento de volumen de los músculos de las pantorrillas (2) (3).

De acuerdo a recientes clasificaciones se han descrito dentro de este cuadro, por lo menos otras dos formas: el tipo menos severo, también ligado al sexo, de comienzo tardío y larga sobrevida, llamado de Becker y una forma igual al tipo Pseudohipertrófico, pero que muestra una fuerte tendencia a ser heredado de manera autosómica recesiva (2) y ser más lentamente progresivo (3).

La frecuencia de la enfermedad no ha sido definitivamente estudiada pero las estimaciones efectuadas, revelan una prevalencia de 66 enfermos vivos por 1 millón de hombres vivos y una incidencia de 279 pacientes por 1 millón de recién nacidos varones (4). Aplicando este criterio para nuestro país deberíamos obtener una prevalencia de 330 casos y una incidencia de 35 enfermos por año (5).

Es sabido que el tratamiento es sólo paliativo, de aquí que la orientación genética de los familiares es por el momento la única alternativa para disminuir la incidencia del cuadro mediante el consejo genético.

Numerosas investigaciones han sido emprendidas con el fin de determinar por medio de diversas técnicas a los portadores: electromiografía, biopsia muscular, isoenzimas de la dehidrogenasa láctica muscular, creatinina, inmunoelectroforesis del suero, electrocardiograma, transaminasas, al-

\footnotetext{
* Instituto de Rehabilitación Infantil.

* Servicio de Neurologfa y Psiquiatría Infantil. Hospital Luis Calvo Mackenna.

«** Departamento de Nutrición y Tecnología de los Alimentos. Sede Sur U. de Chile.
}

dolasa, rubidio marcado, creatinafosfoquinasa (CPK), etc. (3) (6).

Pareciera no haber dudas que la determinación de la CPK en sangre es el método más sensitivo y específico para demostrar portadores heterozigotos. (1) (2) (3) (7) (8) (9) (10) (11) (12).

Existen sin embargo otros cuadros en los cuales esta enzima está anormalmente alta: infarto del miocardio (13), hipotiroidismo, coma hepático, accidente vascular cerebral, polimiositis (14), tetanos (15) y últimamente en la esclerosis lateral amiotrófica (16).

Hay algunas variables cuya importancia relativa en la determinación de la CPK no están claramente definidas y podrían influir en el título de la enzima. Se han mencionado, la edad del portador, su estado hormonal, dieta, ejercicio físico previo al examen (1) (2) (12) (17) (18).

En todos los estudios, sin embargo, una pequeña cantidad $(1 / 4$ a $1 / 3,25$ a $35 \%)$ de todos los heterozigotos examinados, tienen CPK dentro de límites normales. Este hecho, la presencia de falsos negativos es la limitante mayor en la utilidad de la enzima, para proporcionar un consejo genético. (1) (2) (3) (9).

Es nuestro deseo en esta comunicación, relatar nuestra experiencia con 24 familias en las que había por lo menos un miembro con DMP, analizando 47 parientes de primer grado, con la intención de proporcionar un consejo genético basado en determinaciones químicas.

MATERLAL y METODO. Los sujetos fueron 18 padres, 24 madres, 7 hermanos y 23 hermanas de niños con DMP atendidos durante 1973 en el Instituto de Rehabilitación Infantil de Santiago de Chile. Por diversas razones, no se analizó en todos los casos al grupo familiar completo. Los hermanos y hermanas del enfermo tenían todos 
más de 5 años de edad. El total de análisis fue de 100. Cuatro familiares tenian 2 enfermos $\mathrm{c} / \mathrm{u}$ y los enfermos examinados fueron 28 .

El diagnóstico del caso índice fue hecho clinicamente y confirmado mediante CPK, electromiograma y biopsia muscular en la mayoría. Fueron excluidos aquellos en los cuales se sospechó una forma de Becker, el tipo autosómico recesivo u otra miopatía.

Nosotros hemos designado como "portadora conocida" a madres con dos o más hijos enfermos; o 1 hijo enfermo más otro pariente de la línea materna que tenga o haya tenido DMP; o 1 hijo DMP, cuya hermana tiene un hijo con la enfermedad.

Hemos designado como "portadora posible" a una madre o hermana de un caso aislado (1) (2) (9)

Para efectuar el estudio enzimático las familias fueron llevadas en vehículos particulares hasta el laboratorio y no se les exigió preparación previa.

La determinación de CPK, se hizo según el método colométrico № 661 del Boletín Técnico de Sigma. Según esta técnica se considera normal entre 0 y 12 u Sigma/ml, limítrofe entre 12,5 y 20 u Sigma $/ \mathrm{ml}$ y elevado sobre 20 u Sigma $/ \mathrm{ml}$. Para los efectos de los resultados sólo se consideraron como positivos aquellos informados con 20 u 0 más.

Resultados. En la figura 1 se observa la distribución de las 100 muestras tomadas a los parientes en 24 familias, que nos demuestra claramente las diferencias de los títulos de CPK de hombres y mujeres. Todos los varones sanos examinados exhibieron valores normales, en tanto que los de las mujeres se distribuyeron de manera muy diferente como lo demuestran la figura $1 \mathrm{y}$ la tabla 1.

Con el objeto de pesquisar madres portadoras heterozigotas pero con CPK negativo (CPK-), pre-

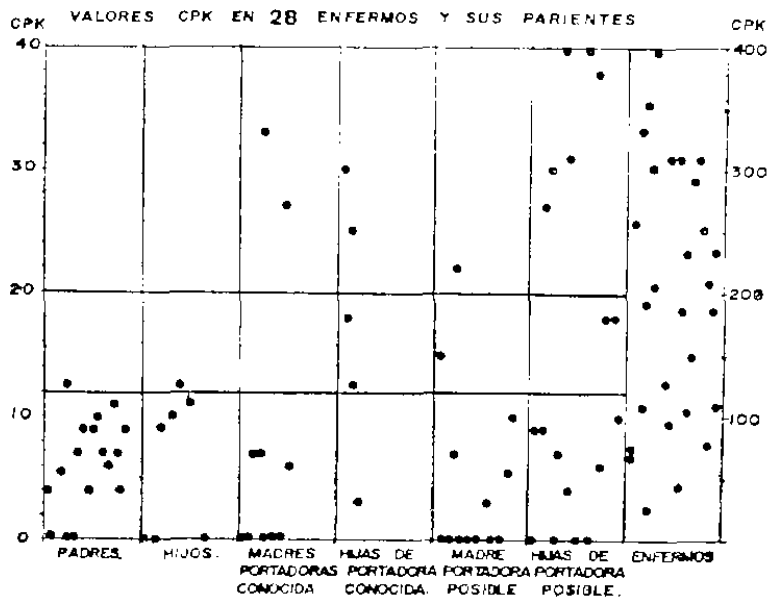

T A B L A 1

VALORES CPK EN MADRES Y HERMANAS

\begin{tabular}{lccc}
\hline & Positivo & Negativo & Total \\
\hline Madres & 3 & 21 & 24 \\
Hermanas & 8 & 15 & 23 \\
\hline To t a I & 11 & 36 & 47 \\
\hline
\end{tabular}

T A B L A No 2

CARACTERISTICAS DE LOS PARIENTES

CPK + CPK- Total

\begin{tabular}{llrr}
\hline Madres portadoras conocidas & 2 & 8 & 10 \\
Hijos de madres portadoras & & & \\
$\quad$ conocidas & $2^{*}$ & 3 & 5 \\
$\begin{array}{l}\text { Madres portadoras posibles } \\
\text { Hijas de madres portadoras }\end{array}$ & 1 & 13 & 14 \\
$\quad$ posibles & $6^{* *}$ & 12 & 18 \\
\hline
\end{tabular}

* Sus madres tienen CPK(-).

** 4 de ellas tienen madres CPK(-).

T A B L A No 3

DETECCION DE PORTADORES POR PEDIGREE Y $C P K$

\begin{tabular}{lccr}
\hline & Pedigree & CPK & Total \\
\hline $\begin{array}{l}\text { Madres portadoras conocidas } \\
\text { Hijas de madres portadoras }\end{array}$ & 10 & $2+(2)$ & 10 \\
$\quad$ conocidas & - & 2 & 5 \\
Madres portadoras posibles & - & $1+(4)$ & 14 \\
Hijas de madres portadoras & & & \\
$\quad$ posibles & - & 6 & 18 \\
( ) Por deducción. & & &
\end{tabular}

sentamos la tabla 2 . Su análisis y su confrontación con el grupo familiar respectivo, nos revela que dos madres portadoras conocidas con CPK-, tienen cada una 1 hija con $\mathrm{CPK}$ anormalmente alto. Esta situación la observamos también para 4 madres portadoras posibles.

Creemos importante destacar que en 7 de las 24 familias estudiadas, no habían hermanas y en 3 existían hermanas pero no fueron examinadas.

En la tabla № 3 hemos comparado la detección de portadoras heterozigotas mediante el estudio del pedigree familiar y la CPK. Se observa 


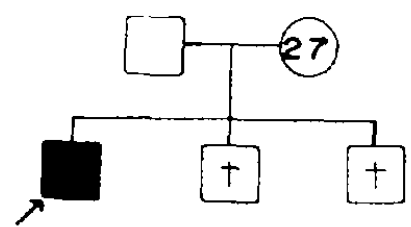

MUESTRA

- PORTADORA CONOCIDA.

- DESASTRE fAMILIAR EN PORTADORA CON CPK +

claramente que 13 de 37 mujeres son diagnosticadas mediante la enzima y que ninguna de éstas pudo haberlo sido mediante el árbol genealógico.

En esa misma tabla, es posible observar que en 24 familias, hemos encontrado 14 que representan casos aislados de DMP. Ahora bien, en 9 de estos 14 grupos familiares se obtuvo CPK- en todos los parientes examinados, Io cual de acuerdo a opiniones generalizadas (2) (9) corresponderían a mutaciones.

COMENTARIO. Los resultados presentados confirman a través del estudio de la CPK, que el mecanismo de herencia está ligado al sexo y que esta enzima puede ser diagnóstico del estado de portador cuando está elevada (1) (2) (3) (7) (8) (9) (15) (19).

Sin embargo (1) (2) (9) (12) (15) es necesario explicar porque no todos los portadores heterozigotos conocidos tienen $\mathrm{CPK}$ alto $(\mathrm{CPK}+)$ y qué importancia tiene para la familia encontrar una mujer con CPK normal o anormal.

El problema de las madres portadoras conocidas. De 10 madres portadoras conocidas obtuvimos 2 con $\mathrm{CPK}+$, pero otras 2 madres CPK-, tienen hijas con la enzima elevada. Según esto a aquellas madres las podemos incluir como heterozigotas, lo cual nos da una positividad del $40 \%$ (1) (2) Fig. 2.

Aunque veremos más adelante las explicacio nes que justifican el $30 \%$ de falsos negativos, la presencia en nuestros casos de un $60 \%$ de negatividad se debe a una muestra estadísticamente muy pequeña.

El problema de las hijas de portadoras conocidas. Según el mecanismo de transmisión recesiva ligado al sexo, deberíamos esperar que el $50 \%$ de las de las portadoras conocidas fueran a su vez portadoras heterozigotas, de ellas y de acuerdo a la positividad de la enzima, el $70 \%$ debería tener CPK. Como fueron examinadas 5 hijas de madres portadoras conocidas, al resultar 2 hijas con enzima anormalmente alta, significa que hemos obtenido para este grupo una positividad de $66,6 \%$ (2 de 3 esperadas), lo cual se adscribe dentro de lo esperado y de lo comunicado por la literatura, (1) (2) (3) (12).

Es importante destacar en este punto, que para el consejo genético de esta clase de portado ras, el estudio del pedigree no es de ninguna utilidad porque él puede sólo señalar probabilidades en un grupo de hermanas pero no determinar exactamente cuál de ellas es la portadora como lo hace la CPK, aunque sea con la limitante de los falsos negativos.

El problema de las madres portadoras posibles $y$ de sus hijas. A diferencia de las familias en que hay portadoras conocidas, en los grupos de portadores posibles es imposible predecir el riesgo de heterozigocidad tanto en las madres como en sus hijas (2) (15). Figuras 3 y 4 .

En estos casos aislados o espontáneos, el estudio enzimático proporciona información adecuada acerca de los heterozigotos.

De acuerdo a nuestros resultados, hemos podido detectar (tabla 4) $11(5+6)$ heterozigotos de 32 exámenes extraídos al grupo que comentamos, a los cuales se les puede dar orientación genética y representa el $34 \%$ de los parientes examinados, porcentaje que está dentro de lo comunicado por otros autores. (1) (2) (9).

Si analizamos lo que ocurre sólo con las madres de casos aislados podemos concluir que nues-

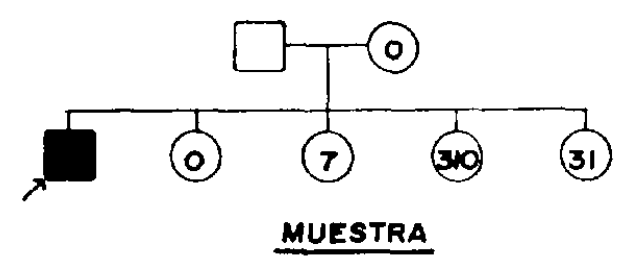

- portadora posiele.
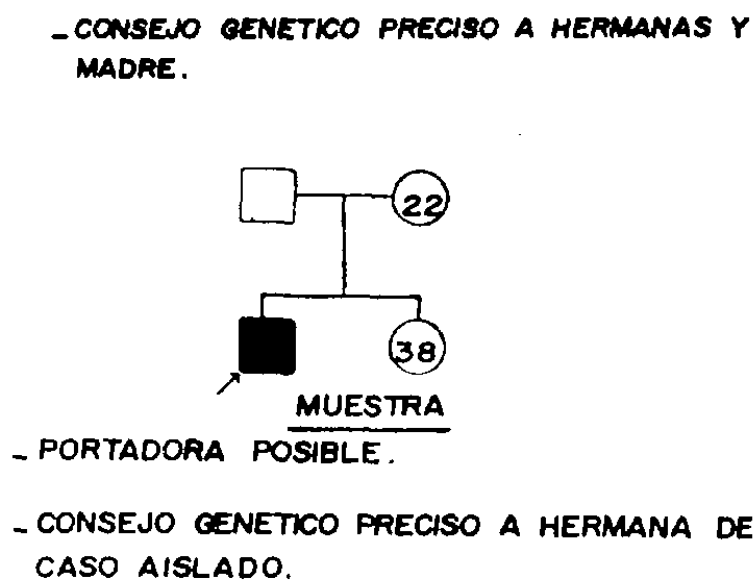
tros resultados, también son semejantes a los observados en la literatura. (1) (2) (9).

De 14 madres portadoras posibles, podemos pesquisar a 5 como heterozigotas ( 1 con $\mathrm{CPK}$ y 4 hijas suyas con $\mathrm{CPK}+$ ). Como estas 5 representan sólo $2 / 3$ de positividad real de la enzima, tendremos que habrán 2 más (para no separar 2,5 madres) heterozigotas, pero falsas negativas. Por lo tanto de 14 habrán 7 portadoras, lo que significa que el riesgo de ser portadora madre en un caso aislado de DMP es del 50\% (2).

El problema de los casos aislados. Ya hemos visto que teóricamente debemos esperar que el $50 \%$ de las madres de casos aislados corresponden a heterozigotos. En la práctica y a través de nuestros resultados, podemos decir que de 14 madres de casos aislados hay 9 en los cuales no fue posible obtener títulos altos de CPK ni en ellas ni en sus hijas. Estas familias bien podrían corresponder al tipo autosómico recesivo, pero a falta de CPK elevado en los padres y ausencia de otros enfermos en la familia que fueran mujeres, esta posibilidad debería ser dejada de lado. Así tendríamos que pensar con Milhorat y Golstone (1) que estos enfermos podrían ser mutantes.

Es, sin embargo, la nuestra una cifra alta, ya que representa el $40 \%$ de 24 familias y el índice de mutaciones comúnmente aceptado es de 24 a $33 \%$ (1) (8) (9). Es posible que esta divergencia esté determinada por las características de la muestra y al número insuficiente de padres examinados.

Otra posibilidad para explicar los casos aislados es el pasaje de genes mutantes a través de mujeres, por generaciones sucesivas sin transmisión sino a los varones en casos aislados. En la mayor parte de los estudios genéticos esta información está limitada debido a las pocas generaciones que es posible estudiar (1).

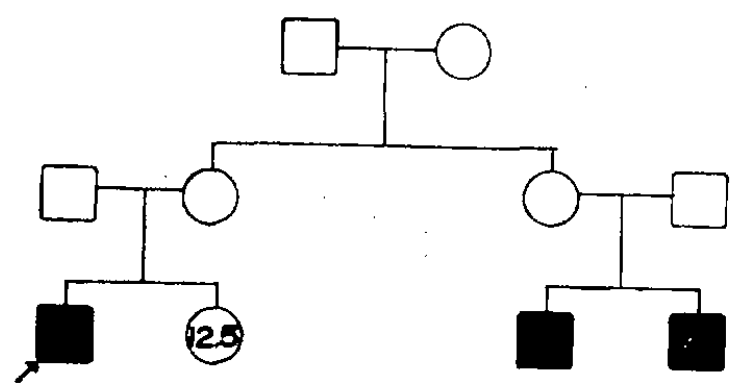

MUESTRA

- OtRo tiPo de PORTADORA CONOCIOA.

- NEFEctividad DE CPK EN LA HERMANA.
El problema de los falsos negativos. Encontrar valores altos de CPK en enfermos y portadores es prácticamente patonogmónico de DMP. La presencia de falsos positivos es de una ocurrencia tan excepcional que CPK en mujeres parientes (madres y hermanas) de enfermos de DMP es un elemento de gran utilidad para detectar heterozigotos (1) (2) (9). Figura 5.

Lo inverso, obtener falsos negativos no indica que el individuo no sea heterozigoto.

Habíamos mencionado que cerca del $1 / 3$ de los portadores heterozigotos son indetectables mediante la CPK. Existen por lo menos cuatro explicaciones al respecto (2):1) Dificultades técnicas, que se rechaza como posibilidad desde el momento que todas las comunicaciones, independientemente de la técnica usada, han dado $100 \%$ de seguridad. 2) Cambios en la edad. También se desecha después que estudios estadísticos revelan que en los portadores, por lo menos, esta relación edad-CPK no existe, 3) Lyonización del cromosoma X. Esta hipótesis afirma que uno de los cromosomas $\mathrm{X}$ de cada célula femenina es inactivo. Este cromosoma viene determinado al azar, por lo que el número de células bajo la influencia de cromosomas $\mathrm{X}$ anormales en portadoras viene también determinado al azar. Por lo tanto las portadoras con predominio de células musculares bajo la influencia de cromosomas $X$ normales serían difíciles de identificar con las actuales técnicas de laboratorio (3) . 4) Desde que algunos autores han planteado la heterogeneidad genética para la DMP, postulando que el cuadro no sería una sola entidad clínica, la variabilidad en pesquisar portadores podría estar en relación a este hecho.

\section{RESUMEN}

1.- Se determinó la creatinafosfoquinasa sanguinea $(C P K)$ en 72 parientes de 24 familias en las que había por lo menos un niño enfermo con Distrofia Muscular Progresiva Pseudohipertrófico tipo Duchenne, con el objeto de detectar portadores heterozigotos para proporcionarles consejo genético.

2.-El 40\% de las "portadoras conocidas" $y$ el $66 \%$ de sus hijas esperadas como portadoras, resultaron ser heterozigotas.

3.- El 34\% de las "madres portadoras posibles" más sus hijas fueron detectadas como heterozigotas.

4.- El riesgo de ser madre portadora heterozigota de un caso aislado de DMP es de $50 \%$. 
5.- Los resultados demuestran que la titulación de la CPK es de gran utilidad para pesquisar parientes heterozigotos de $D M P$.

\section{A G RADECIMIE N T OS}

Los autores agradecen a la Dircción y Personal del Instituto de Rehabilitación Infantil las facilidades otorgadas para la realización de este estudio. Nuestro reconocimiento también para la Sra. M. Teresa Segura por su valiosa cooperación en el aspecto estadistico, y a la Dra. Ana María Pino por su colaboración en la Técnica de CPK.

\section{REFERENCIAS}

1.-Milhorat, A. T., and Goldstone, L.: The carrier state in Muscular Dystrophy of the Duchenne type, JAMA: 194: 130, 1965.

2.-Thompson, M. W., Murphy, M. B. and McAlpine, $P h$. J. An assesment o fthe creatinekinase test in the detection of carrier of Duchenne Muscular Dystrophy, J. Pediat. 71: 82, 1967.

3.- Swaiman, K. F. and Wright. F. S.: Enfermedades neuromusculares en el lactante en el niño. Editorial Pediátrica, Barcelona, 1972.

4.- Morton N. E., and Chung, C. S.: Formal genetics of Muscular Dystrophy, Am. J. Human. Genet. 11: 360, 1959.

5.- Anales del Servicio Nacional de Salud. Santiago Chile, 1972.

6.-Bradley, W. C. Gardner-Medwin. D., Haggith J. Walton J. N. and Hesp. R. Use of Rubidium Chloride $\mathrm{Rb} 86$ in the detection of Duchenne Muscular Dystrophy. Arch. Neurol. 25: 193, 1971.

7.-Smith, H. L. Amick, L. O., and Johnson, W. W.: The carrier state in Muscular Dystrophy, J. Pediat. 69: 67, 1966 .

8.- Carlson, C. B. and Swanson, A. C. Genética en enfermedades neuromusculares, Clin. Pediat. Nov. 1967. Pág. 957.
9.- Hertnarska, $H$. The vallue of determination of Serum creatine phosphokinase activity in detection of carriers of Progresive Muscular Dystrophy, Polish Med. J. 7: 224, 1968.

10.-Niebrosj-Doboz, I. Jedrzejowska, H., and Hetmarska, H.: Blood enzymas in Duchenne's Progressive Muscular Dystrophy and their correlation with the clinical and histological pictures. Acta. Med. Pol. 11: $26,1970$.

11.- Shearin B. R. and Mansuwam, P.: Pseudohypertrophic Muscular Dystrophy, Am. J. Dis. Child. 125: 580, 1973.

12.-Hudgson, P., Gardner Medwin, D., Pennington, R. B. T., and Walton, J. N.: Studies of the carrier state in the Duchenna type of Muscular Dystrophy, J. neurol. Neurosurg. Psychiat. 30: 416, 1967.

13.-Sobel, B, E.: Creatinephosphokinase and myocardial infartation, JAMA. 229: 201, 1974.

14.- Schiavone, D. J. and Kaldor, J.: Creatinephosphokinase levels and cerebral disease, Med. J. Aust. 2: $790,1965$.

15.-Kelly, S., Kelly, W., and Swift. H. L.: Serum creatinephosphokinase in 4 generations of a muscular Dystrophy family, Am. J. Clin. Path. 45: 377, 1966.

16.- Achari, A. N., and Anderson, M. S.: Creatinephosphokinase in Amiotrophic lateral sclerosis, Neurology 24: 834, 1974.

17.-Canari, A. González, M., and Semeniuk, G. B.: Increased levels of Serum-creatinephosphokinase after transient Limb Ischaemia in patients with Muscular Dystrophy, Lancet. 31. JAN., 1970.

18.-Munsat, T. L., Baloch, R., Pearson, C. M., and Fonojer, W.: Serum enzyme alterations in Neuromuscular Disorders, JAMA 226: 1536, 1973.

19.- Murphy, E. C., and Waisburg, H.: Neuromuscular disorders in children, Pediat. Clin. N. A. 21: 917. 1974. 